\title{
Stochastic monotonicity and duality for one-dimensional Markov processes (revised)
}

\author{
Vassili N. Kolokoltsov* \\ September 18, 2018
}

\begin{abstract}
The theory of monotonicity and duality is developed for general one-dimensional Feller processes, extending the approach from [10]. Moreover it is shown that local monotonicity conditions (conditions on the Lévy kernel) are sufficient to prove the well-posedness of the corresponding Markov semigroup and process, including unbounded coefficients and processes on the half-line.
\end{abstract}

Key words. Stochastic monotonicity, duality, one-dimensional Markov processes, LévyKchintchine type generators.

\section{Introduction}

A Markov process $X_{t}$ in $\mathbf{R}$ is called stochastically monotone if the function $\mathbf{P}\left(X_{t}^{x} \geq y\right)$ (as usual, $x$ stands for the initial point here) is nondecreasing in $x$ for any $y \in \mathbf{R}, t \in \mathbf{R}_{+}$, or, equivalently (by linearity and approximation), if the corresponding Markov semigroup preserves the set of non-decreasing functions. A Markov process $Y_{t}$ in $\mathbf{R}$ is called dual to $X_{t}$ if

$$
\mathbf{P}\left(Y_{t}^{y} \leq x\right)=\mathbf{P}\left(X_{t}^{x} \geq y\right)
$$

for all $t>0, x, y \in \mathbf{R}$. If a dual Markov process exists it is obviously unique.

Stochastic monotonicity for Markov chains is well studied and applied for the analysis of many practical models, see e.g. [1], [5], [6], [16]. Stochastic monotonicity and the related duality are also well studied for diffusions (see [9] and [4]) and jump-type Markov processes (see [1], 22, [3]), [20]). In [18] the monotonicity for stable processes was analyzed. For general Markov processes the analysis of stochastic monotonicity and related duality was initiated in [10] devoted to the case of one dimensional processes with polynomial coefficients (note some nasty typos in the expression of the dual generator in [10]). This was related to interacting particle models (see also [15]), and related Markov models in financial mathematics. In [19] the theory of monotonicity was extended to multidimensional processes of Lévy-Khintchine type with Lévy measures having a finite first internal moment, i.e. with $\int_{|y|<1} \nu(x, d y)<\infty$ (slightly more general in fact).

${ }^{*}$ Department of Statistics, University of Warwick, Coventry CV4 7AL UK, Email: v.kolokoltsov@warwick.ac.uk 
In this note we first extend the theory of monotonicity and duality to arbitrary onedimensional Feller processes (Sections 2 and 3), following approach from [10]. We shall give a criterion of stochastic monotonicity in terms of the generator of $X_{t}$ and, under additional regularity assumptions, the explicit formula for the dual generator. Here our approach is based on the discretization and eventually on the theory of stochastic monotonicity for Markov chains.

In the second part of the paper (Sections 4,5) we use an alternative approach to the analysis of monotonicity, adapting in particular the method used in [13] for the generators of order at most one. Most importantly we show that local monotonicity conditions (conditions on the Lévy kernel) are sufficient to prove the well-posedness of the corresponding Markov semigroup and process, thus contributing to the important problem of building a process from a given pre-generator (see e.g. [8], [11], [14]). Stochastically monotone processes on the half-line are finally constructed.

Most of the results given are extendable to arbitrary dimensions, but the exposition of one-dimensional theory as a first step seems to be in order, not least because its relevance to option pricing, see [17].

To conclude the introduction, let me thank professor $\mathrm{Mu} F \mathrm{Fa}$ Chen for bringing to my attention some relevant recent publications of the Chinese school.

\section{Monotonicity via discrete approximations}

Let us recall shortly the theory of stochastic monotonicity for Markov chains, following [1. Recall first that an infinitesimally stochastic matrix or $Q$-matrix $Q=\left(Q_{m n}\right)$ with $m, n \in \mathbf{Z}$ is such a matrix that $Q_{n m} \geq 0$ for $m \neq n$ and

$$
Q_{n n}=-\sum_{m \neq n} Q_{n m}
$$

for all $n$. To any such matrix there corresponds a Markov process (generally not unique) with the generator given by the matrix $Q$ (which we shall denote by the same letter):

$$
(Q f)_{n}=\sum_{m} Q_{n m} f_{m}
$$

Taking into account the properties of $Q$, one can rewrite it in other two useful forms:

$$
(Q f)_{n}=\sum_{m \neq n} Q_{n m}\left(f_{m}-f_{n}\right)=\sum_{m} Q_{n m}\left(f_{m}-f_{n}\right)
$$

If the intensity of jumps, specified $Q$ is uniformly bounded, that is

$$
\sup _{n}\left|Q_{n n}\right|<\infty
$$

the corresponding Markov process is unique and conservative, the latter meaning that the corresponding semigroup preserves constants.

A $Q$-matrix is called stochastically monotone if

$$
\sum_{j \geq l} Q_{n j} \leq \sum_{j \geq l} Q_{n+1, j} \quad \forall l \neq n+1
$$


(we separate indices by commas, like $Q_{n, m}$, if needed for clearness). The key discrete result states (proof in [1]) that if $Q$ is stochastically monotone, then the corresponding Markov chain $X_{t}$ is stochastically monotone in the sense that $\mathbf{P}\left(X_{t}^{n} \geq m\right)$ is nondecreasing in $n$ for any $m, t$ and the dual Markov chain $Y_{t}$ (satisfying (1) with integer $x, y$ ) has the $Q$-matrix

$$
\tilde{Q}_{n j}=\sum_{l=n}^{\infty}\left(Q_{j l}-Q_{j-1, l}\right) .
$$

It turns out that the monotonicity condition becomes more transparent if written in terms of the matrix $\omega=\left(\omega_{n m}\right)$, which is connected with the $Q$-matrix by the equation $\omega_{n m}=Q_{n n+m}$. Thus the entries $\omega_{n m}$ define the probabilities of jumps to the right $(m>0)$ and to the left $(m<0)$ of $n$. In fact, condition (3) takes form

$$
\sum_{m \geq l-n} \omega_{n m} \leq \sum_{m \geq l-n-1} \omega_{n+1, m} \quad \forall l \neq n+1
$$

and this condition is equivalent to two separate conditions on the right and the left jumps:

$$
\begin{array}{cl}
\sum_{m \geq k} \omega_{n m} \leq \omega_{n+1, k-1}+\sum_{m \geq k} \omega_{n+1, m} \quad \forall k \geq 2, \\
\omega_{n,-k+1}+\sum_{m \geq k} \omega_{n,-m} \geq \sum_{m \geq k} \omega_{n+1,-m} \quad \forall k \geq 2 .
\end{array}
$$

Remark. It is straightforward to see that (6) is equivalent to (5) for $l \geq n+2$. equation (2) implies that (7) is equivalent to (3) for $l \leq n$.

It is worth noting that (6), (17) are satisfied if $\omega_{n, 1}, \omega_{n,-1}$ are arbitrary (non-negative) and other coefficients satisfy simpler inequalities

$$
\sum_{m \geq k} \omega_{n m} \leq \sum_{m \geq k} \omega_{n+1, m}, \quad \sum_{m \geq k} \omega_{n,-m} \geq \sum_{m \geq k} \omega_{n+1,-m} \quad \forall k \geq 2 .
$$

Finally equation (4) rewrites as

$$
\tilde{Q}_{n, n+i}=\sum_{l=-i}^{\infty}\left(Q_{n+i, n+i+l}-Q_{n+i-1, n+i+l}\right)
$$

and hence in terms of $\tilde{\omega}_{n m}=\tilde{Q}_{n, n+m}$ as

$$
\tilde{\omega}_{n i}=\sum_{l=-i}^{\infty}\left(\omega_{n+i, l}-\omega_{n+i-1, l+1}\right) .
$$

In particular, if $\omega_{n m}$ do not vanish only for $|m| \leq 1$, the same holds for $\tilde{\omega}$ and

$$
\tilde{\omega}_{n 1}=\omega_{n,-1}, \quad \tilde{\omega}_{n,-1}=\omega_{n-1,1}
$$

Moreover, by duality, right jumps turn to the left jumps and vice versa, i.e. if $\omega_{n,-i}=0$ for all $i>0$, then $\tilde{\omega}_{n i}=0$ for $i>0$ and

$$
\tilde{\omega}_{n,-i}=\omega_{n-i, i}+\sum_{l=i+1}^{\infty}\left(\omega_{n-i, l}-\omega_{n-i-1, l}\right) \quad i>0
$$


and if $\omega_{n, i}=0$ for all $i>0$, then $\tilde{\omega}_{n,-i}=0$ for $i>0$ and

$$
\tilde{\omega}_{n, i}=\omega_{n+i-1,-i}+\sum_{l=i+1}^{\infty}\left(\omega_{n+i-1,-l}-\omega_{n+i,-l}\right) \quad i>0 .
$$

The following is the main result of this short paper.

Theorem 2.1. Let $X_{t}$ be a Feller process in $\mathbf{R}$ with the generator of the usual LévyKchintchine form

$$
L f(x)=\frac{1}{2} G(x) f^{\prime \prime}(x)+b(x) f^{\prime}(x)+\int\left(f(x+y)-f(x)-f^{\prime}(x) y \mathbf{1}_{B_{1}}(y)\right) \nu(x, d y)
$$

with continuous $G, b, \nu$, and let the space $C_{c}^{2}(\mathbf{R})$ be a core. For simplicity assume also (though this is not very essential) that the coefficients are bounded, that is

$$
\sup _{x}\left(G(x)+|b(x)|+\int\left(1 \wedge y^{2}\right) \nu(x, d y)\right)<\infty .
$$

If the Lévy measures $\nu$ are such that for any $a>0$ the functions

$$
\int_{a}^{\infty} \nu(x, d y), \quad \int_{-\infty}^{-a} \nu(x, d y)
$$

are non-decreasing and non-increasing respectively, as functions of $x$, then the process $X_{t}$ is stochastically monotone. Moreover, the dual Markov process exists.

Proof. Let $h>0$ and set

$$
\begin{aligned}
& L_{h} f(x)=G(x) \frac{f(x+h)+f(x-h)-2 f(x)}{2 h^{2}}+|b(x)| \frac{f(x+h \operatorname{sgn}(\mathrm{b}(\mathrm{x})))-\mathrm{f}(\mathrm{x})}{h} \\
& +\sum_{m=1}^{\infty}\left[f(x+m h)-f(x)+\frac{f(x-h)-f(x)}{h} m h \mathbf{1}_{B_{1}}(m h)\right] \nu(x,[m h, m h+h)) \\
& +\sum_{m=1}^{\infty}\left[f(x-m h)-f(x)+\frac{f(x+h)-f(x)}{h} m h \mathbf{1}_{B_{1}}(m h)\right] \nu(x,(m h-h, m h]) .
\end{aligned}
$$

These operators approximate $L$ on $C_{c}^{2}(\mathbf{R})$ for $h \rightarrow 0$. Since this space is a core for $L$, the corresponding semigroups converge. But by the above mentioned result for Markov chains, the processes $X_{t, h}$ generated by $L_{h}$ are stochastically monotone. Consequently the same holds for the process $X_{t}$ generated by $L$. Again by the properties of Markov chains, the dual processes $Y_{t, h}$ to $X_{t, h}$ are well defined. Their transition probabilities converge, because they are expressed in terms of the converging transition probabilities of $X_{t, h}$. The limiting Markov process $Y_{t}$ is dual to $X_{t}$. 


\section{Dual generators}

Under some regularity assumptions we can write explicitly the generator of the dual process. To simplify formulas, we shall do it only for Lévy measures supported on $\mathbf{R}_{+}$ (the case of measures supported on $\mathbf{R}_{-}$is symmetric and is done using equation (12) instead of (11) used below).

Proposition 3.1. Under the assumptions of the above Theorem suppose additionally that the Lévy measures are supported on $\mathbf{R}_{+}$and either $(i) \nu(x, d y)=\nu(x, y) d y$ with $\nu(x, y)$ differentiable in $x$, or (ii) $\nu(x, d y)=a(x) \nu(d y)$ with a certain Lévy measure $\nu$ and a continuously differentiable function a (decomposable generator case). Then the generator of the dual Markov process acts by

$$
\begin{gathered}
L f(x)=\frac{1}{2} G(x) f^{\prime \prime}(x)-\left[\frac{1}{2} G^{\prime}(x)+b(x)\right] f^{\prime}(x) \\
+\int_{0}^{\infty}\left[f(x-y)-f(x)+f^{\prime}(x) \mathbf{1}_{B_{1}}(y)\right] \tilde{\nu}(x, d y)+f^{\prime}(x) \int_{0}^{1} y(\nu-\tilde{\nu})(x, d y)
\end{gathered}
$$

on $C_{c}^{2}(\mathbf{R})$, where

$$
\tilde{\nu}(x, d y)=\left[\nu(x-y, y)+\frac{\partial}{\partial x} \int_{y}^{\infty} \nu(x-y, z) d z\right] d y
$$

in case (i) and

$$
\tilde{\nu}(x, d y)=a(x-y) \nu(d y)+a^{\prime}(x-y) \int_{y}^{\infty} \nu(d z) d y
$$

in case (ii).

Proof. By linearity one can calculate the dual generator separately for diffusive, drift and integral parts of $L$. By (10) the dual generator corresponding to the first term in (15) has the form

$$
\left(2 h^{2}\right)^{-1}[G(x) f(x+h)+G(x-h) f(x-h)-(G(x)+G(x-h)) f(x)],
$$

which converges to

$$
\frac{1}{2}\left[G(x) f^{\prime \prime}(x)-G^{\prime}(x) f^{\prime}(x)\right]
$$

as $h \rightarrow 0$. Similarly analyzing the drift part yields the first two terms in (16)).

Next, from equation (11), it follows that the dual operator to the first sum in of (15) equals

$$
\begin{gathered}
\sum_{m=1}^{\infty}(f(x-m h)-f(x))[\nu(x-m h,[m h, m h+h)) \\
\left.+\sum_{l=m+1}^{\infty} \nu(x-m h,[l h, l h+h))-\nu(x-m h-h,[l h, l h+h))\right] \\
+\frac{f(x+h)-f(x)}{h} \sum_{m=1}^{\infty} m h \mathbf{1}_{B_{1}}(m h) \nu(x,[m h, m h+h)) .
\end{gathered}
$$


In case (i) it rewrites as

$$
\begin{gathered}
\sum_{m=1}^{\infty}(f(x-m h)-f(x))\left[\int_{m h}^{m h+h} \nu(x-m h, y) d y+\sum_{l=m+1}^{\infty} \int_{l h}^{l h+h}(\nu(x-m h, z)-\nu(x-m h-h, z)) d z\right] \\
+\frac{f(x+h)-f(x)}{h} \sum_{m=1}^{\infty} m h \mathbf{1}_{B_{1}}(m h) \int_{m h}^{m h+h} \nu(x, y) d y
\end{gathered}
$$

yielding the first formula for $\tilde{\nu}$. In case (ii), it rewrites as

$$
\begin{aligned}
\sum_{m=1}^{\infty}(f(x-m h)- & f(x))\left[a(x-m h) \nu([m h, m h+h))+\sum_{l=m+1}^{\infty}(a(x-m h)-a(x-m h-h) \nu([l h, l h+h))]\right. \\
& +\frac{f(x+h)-f(x)}{h} \sum_{m=1}^{\infty} m h \mathbf{1}_{B_{1}}(m h) a(x) \nu([m h, m h+h)) d y,
\end{aligned}
$$

yielding the second one.

\section{Well-posedness via monotonicity}

Apart from the definition of monotonicity, the remaining exposition is independent of the previous results. Let us first describe our approach in the simplest situation.

Theorem 4.1. Let

$$
L f(x)=\int\left(f(x+y)-f(x)-f^{\prime}(x) y\right) \nu(x, d y)
$$

with a continuous Lévy kernel $\nu$ such that

$$
\sup _{x} \int\left(|y| \wedge|y|^{2}\right) \nu(x, d y)<\infty
$$

and the first two derivatives $\nu^{\prime}(x, d y)$ and $\nu^{\prime}(x, d y)$ of $\nu$ with respect to $x$ exist weakly and define continuous signed Lévy kernels such that

$$
\sup _{x} \int\left(|y| \wedge|y|^{2}\right)\left|\nu^{\prime}(x, d y)\right|<\infty, \quad \sup _{x} \int\left(|y| \wedge|y|^{2}\right)\left|\nu^{\prime \prime}(x, d y)\right|<\infty
$$

If for any $a>0$ the functions

$$
\int_{a}^{\infty} \nu(x, d y), \quad \int_{-\infty}^{-a} \nu(x, d y)
$$

are non-decreasing and non-increasing respectively, then $L$ generates a unique Feller semigroup with the generator given by (17) on the subspace $C_{\infty}(\mathbf{R}) \cap C^{2}(\mathbf{R})$. The corresponding process is stochastically monotone. 
Proof. We shall use the following two Taylor formulas:

$$
f(x+y)-f(x)-f^{\prime}(x) y=\int_{0}^{y}\left(f^{\prime}(x+z)-f^{\prime}(x)\right) d z=\int_{0}^{y}(y-z) f^{\prime \prime}(x+z) d z,
$$

where of course $\int_{0}^{y}=-\int_{y}^{0}$ for $y<0$.

Differentiating the equation $\dot{f}=L f$ with respect to the spatial variable $x$ yields the following equation for $g(x)=f^{\prime}(x)$ :

$$
\frac{d}{d t} g(x)=(L+K) g(x)
$$

where

$$
\begin{gathered}
K g(x)=\int\left(f(x+y)-f(x)-f^{\prime}(x) y\right) \nu^{\prime}(x, d y) \\
=\int_{0}^{\infty}\left(\int_{0}^{y}(g(x+z)-g(x)) d z\right) \nu^{\prime}(x, d y)-\int_{-\infty}^{0}\left(\int_{y}^{0}(g(x+z)-g(x)) d z\right) \nu^{\prime}(x, d y) \\
=\int_{0}^{\infty} d z(g(x+z)-g(x)) \int_{z}^{\infty} \nu^{\prime}(x, d y)-\int_{0}^{\infty} d z(g(x-z)-g(x)) \int_{-\infty}^{-z} \nu^{\prime}(x, d y) .
\end{gathered}
$$

The main observation is that by the assumptions of the theorem both terms represent conditionally positive operators of the Lévy-Khintchine type. Differentiating once more one gets for $v=g^{\prime}=f^{\prime \prime}$ the equation

$$
\begin{gathered}
\frac{d}{d t} v(x)=(L+2 K) v(x)+\int\left(f(x+y)-f(x)-f^{\prime}(x) y\right) \nu^{\prime \prime}(x, d y), \\
=(L+2 K) v(x)+\int_{-\infty}^{\infty}\left[\mathbf{1}_{|y| \leq 1} \int_{0}^{y}(y-z) v(x+z) d z+\mathbf{1}_{|y|>1} \int_{0}^{y}(g(x+z)-g(x)) d z\right] \nu^{\prime \prime}(x, d y),
\end{gathered}
$$

and the last term represents a sum of a bounded operator applied to $v$ and a bounded curve whenever $g$ is bounded.

To make the rigorous analysis let us introduce the approximating operator $L_{h}, h>0$, as

$$
L_{h} f(x)=\int_{|y|>h}\left(f(x+y)-f(x)-f^{\prime}(x) y\right) \nu(x, d y) .
$$

Then $L_{h}$ is the sum of the first order operator and a bounded operator in $C_{\infty}(\mathbf{R})$ (the latter is due to our assumptions on the moment of $\nu$ ). Hence it generates a conservative Feller semigroup $T_{t}^{h}$ for any $h>0$. By the form of the equations for $f^{\prime}$, i.e. $\dot{g}=\left(L_{h}+K_{h}\right) g$ with bounded (in $C(\mathbf{R})$ ) and conditionally positive $K_{h}$, and also $f^{\prime \prime}$, one concludes that this semigroup acts by positive contractions on the derivatives $g=f^{\prime}$ and by bounded operators on $v=f^{\prime \prime}$ uniformly in $h$. Hence the spaces $C_{\infty}(\mathbf{R}) \cap C^{1}(\mathbf{R})$ and $C_{\infty}(\mathbf{R}) \cap C^{2}(\mathbf{R})$ are both invariant under $T_{h}^{t}$. Moreover, for any $f \in C_{\infty}(\mathbf{R}) \cap C^{2}(\mathbf{R})$, the functions $T_{h}^{t} f$ belong to $C_{\infty}(\mathbf{R}) \cap C^{2}(\mathbf{R})$ with bounds uniform in $h \in(0,1]$ and $t \in\left[0, t_{0}\right]$ for any $t_{0}$.

Therefore, writing

$$
\left(T_{t}^{h_{1}}-T_{t}^{h_{2}}\right) f=\int_{0}^{t} T_{t-s}^{h_{2}}\left(L_{h_{1}}-L_{h_{2}}\right) T_{s}^{h_{1}} d s
$$


for arbitrary $h_{1}>h_{2}$ and estimating

$$
\left|\left(L_{h_{1}}-L_{h_{2}}\right) T_{s}^{h_{1}} f(x)\right| \leq \int_{B_{h_{1}}}\left\|T_{s}^{h_{1}} f\right\|_{C_{2}(\mathbf{R})}|y|^{2} \nu(x, d y)=o(1)\|f\|_{C^{2}}(\mathbf{R}), \quad h_{1} \rightarrow 0,
$$

yields

$$
\left\|\left(T_{t}^{h_{1}}-T_{t}^{h_{2}}\right) f\right\|=o(1) t\|f\|_{C^{2}}(\mathbf{R}), \quad h_{1} \rightarrow 0 .
$$

Therefore the family $T_{t}^{h} f$ converges to a family $T_{t} f$, as $h \rightarrow 0$. Clearly the limiting family $T_{t}$ specifies a strongly continuous semigroup in $C_{\infty}(\mathbf{R})$.

Applying to $T_{t}$ the same procedure, as was applied above to $T_{t}^{\epsilon}$ (differentiating the evolution equation with respect to $x$ ), shows that $T_{t}$ defines also a contraction semigroup in $C_{\infty}(\mathbf{R}) \cap C^{1}(\mathbf{R})$, preserving positivity of derivatives (and hence stochastically monotone) and a bounded semigroup in $C_{\infty}(\mathbf{R}) \cap C^{2}(\mathbf{R})$.

Writing

$$
\frac{T_{t} f-f}{t}=\frac{\left(T_{t}-T_{t}^{\epsilon}\right) f}{t}+\frac{T_{t}^{\epsilon} f-f}{t}
$$

and noting that by (20) the first term is of order $o(1)\|f\|_{C^{2}}$ as $h \rightarrow 0$ allows to conclude that

$$
\lim _{t \rightarrow 0} \frac{T_{t} f-f}{t}=L f
$$

for any $f \in C_{\infty}(\mathbf{R}) \cap C^{2}(\mathbf{R})$. Hence for these $f$, the semigroup $T_{t} f$ provides classical solutions to the Cauchy problem $\dot{f}=L f$. By the standard duality argument this implies the required uniqueness.

Let us discuss a more general situation including unbounded coefficients, where we include a separate term in the generator to handle in a unified way a simpler situation of Lévy measures with a finite first moment. Let $C_{\infty,|.|}(\mathbf{R})$ denote the Banach space of continuous functions $g$ on $\mathbf{R}$ such that $\lim _{x \rightarrow \infty} g(x) /|x|=0$, equipped with the norm $\|g\|_{C_{\infty,|.|}}=\sup _{x}(|g(x)| /(1+|x|))$.

Theorem 4.2. Let for the operator

$$
\begin{gathered}
L f(x)=\frac{1}{2} G(x) f^{\prime \prime}(x)+b(x) f^{\prime}(x) \\
+\int\left(f(x+y)-f(x)-f^{\prime}(x) y\right) \nu(x, d y)+\int(f(x+y)-f(x)) \mu(x, d y),
\end{gathered}
$$

the following conditions hold:

(i) The functions $G(x)$ and $b(x)$ are twice continuously differentiable, $G$ is nonnegative, and the first two derivatives of $\nu$ and $\mu$ with respect to $x$ exists weakly as signed Borel measures and are continuous in the sense that the integral

$$
\int f(y)\left(\nu(x, d y)+\left|\nu^{\prime}(x, d y)\right|+\left|\nu^{\prime \prime}(x, d y)\right|\right)
$$

is bounded and depends continuously on $x$ for any continuous $f(y) \leq|y| \wedge|y|^{2}$ and the integral

$$
\int f(y)\left(\mu(x, d y)+\left|\mu^{\prime}(x, d y)\right|+\left|\mu^{\prime \prime}(x, d y)\right|\right)
$$


is bounded and depends continuously on $x$ for any continuous $f(y) \leq|y|$.

(ii) For any $a>0$ the functions

$$
\int_{a}^{\infty} \nu(x, d y), \quad \int_{-\infty}^{-a} \nu(x, d y)
$$

are non-decreasing and non-increasing respectively.

(iii) For a constant $c>0$

$$
\begin{aligned}
& b(x)+\int|y|\left(\mu(x, d y)+\int_{-\infty}^{-x}|y+x| \nu(d y) \leq c(1+x), \quad x>1,\right. \\
& -b(x)+\int|y|\left(\mu(x, d y)+\int_{-x}^{\infty}|y+x| \nu(d y) \leq c(1+|x|), \quad x<-1 .\right.
\end{aligned}
$$

Then the martingale problem for $L$ in $C_{c}^{2}(\mathbf{R})$ is well posed, the corresponding process $X_{t}^{x}$ is strong Markov and such that

$$
\mathbf{E}\left|X_{t}^{x}\right| \leq e^{c t}(|x|+c),
$$

its contraction Markov semigroup preserves $C(\mathbf{R})$ and extends from $C(\mathbf{R})$ to a strongly continuous semigroup in $C_{\infty,|.|}(\mathbf{R})$ with a domain containing $C_{c}^{2}(\mathbf{R})$. If additionally, for any $a>0$ the functions

$$
\int_{a}^{\infty} \mu(x, d y), \quad \int_{-\infty}^{-a} \mu(x, d y)
$$

are non-decreasing and non-increasing respectively, then the process $X_{t}^{x}$ is stochastically monotone.

Proof. By condition (23) and the method of Lyapunov function (see e.g. Section 5.2 in [13]) with the Lyapunov function $f_{L}$ being a regularized absolute value, i.e. $f_{L}(x)$ is twice continuously differentiable positive convex function coinciding with $|x|$ for $|x|>1$, the theorem is reduced to the case of bounded coefficients. And in this case its proof is a straightforward extension of Theorem 4.1, where the approximating operator is now

$$
\begin{gathered}
L_{h} f(x)=\frac{1}{2} G(x) f^{\prime \prime}(x)+b(x) f^{\prime}(x) \\
+\int_{|y|>h}\left(f(x+y)-f(x)-f^{\prime}(x) y\right) \nu(x, d y)+\int_{|y|>h}(f(x+y)-f(x)) \mu(x, d y),
\end{gathered}
$$

and the rest of the proof remains the same, if one also takes into account that the diffusion part of this $L$ generates a Feller semigroup due to the well known fact about diffusions with Lipschitz coefficients.

\section{Processes on the half-line}

Theorem 5.1. Let an operator $L$ be given by (21) for $x>0$ and the following conditions hold:

(i) The supports of measures $\nu(x,$.$) and \mu(x,$.$) belong to \mathbf{R}_{+}=\{x>0\}$,

$$
\sup _{x \in(0,1]}\left[|b(x)|+G(x)+\int|y| \mu(x, d y)+\int\left(y \wedge y^{2}\right) \nu(x, d y)\right]<\infty,
$$


and the condition (i) of Theorem 4.2 holds for $x>0$.

(ii) For any $a>0$ the functions

$$
\int_{a}^{\infty} \nu(x, d y), \quad \int_{a}^{\infty} \mu(x, d y)
$$

are non-decreasing in $x$.

(iii) For a constant $c>0$

$$
b(x)+\int y \mu(x, d y) \leq c(1+x), \quad x>1
$$

Then the stopped martingale problem for $L$ in $C_{c}^{2}(\mathbf{R})$ is well posed and specifies a stochastically monotone Markov process $X_{t}^{x}$ in $\overline{\mathbf{R}}_{+}=\{x \geq 0\}$.

Proof. It follows from Theorem 4.2 and the well known localization procedure (see e.g. [7]) for martingale problems.

Applying the results from [11] on the boundary points of jump-type processes, one can directly deduce from Theorem 5.1 various regularity properties of the stopped process and its semigroup (extending the results from [10] obtained there under restrictive technical assumptions). For example, one obtains the following.

Corollary 1. Let the assumptions of Theorem 5.1 hold.

(i) Suppose

$$
G(x)=O\left(x^{2}\right), \quad \int_{0}^{1} z^{2} \nu(x, d z)=O\left(x^{2}\right), \quad|b(x) \wedge 0|=O(x),
$$

for $x \rightarrow 0$, then the point $x$ is inaccessible for $X_{t}^{x}$ and its semigroup preserves the space $C\left(\mathbf{R}_{+}\right)$of bounded continuous functions on $\mathbf{R}_{+}$.

(ii) Suppose that $\lim _{x \rightarrow 0} b(x)$ exists and $G(x)=\alpha x(1+o(1))$ as $x \rightarrow 0$ with a constant $\alpha>0$. If $\alpha<b(0)$, then again the point $x$ is inaccessible for $X_{t}^{x}$ and its semigroup preserves the space $C\left(\mathbf{R}_{+}\right)$. If $\alpha>b(0)$, then the boundary point $x$ is t-regular for $X_{t}^{x}$ and its semigroup preserves the space $C\left(\overline{\mathbf{R}}_{+}\right)$of bounded continuous functions on $\overline{\mathbf{R}}_{+}$.

\section{References}

[1] W.J. Anderson. Continuous - Time Markov Chains. Probability and its Applications. Springer Series in Statistics. Springer 1991.

[2] A. Chen and H. Zhang. Stochastic monotonicity and duality for continuous time Markov chains with general Q-matrix. Southeast Asian Bull. Math. 23:3 (1999), 383-408.

[3] M.F. Chen. From Markov Chains to Non-Equilibrium Particle Systems. World Scientific, Singapore, 2 nd Ed., 2004.

[4] M.F. Chen, F.Y. Wang. On order-preservation and positive correlations for multidimensional diffusion process. Prob. Th. Rel. Fields 95 (1993), 421-428. 
[5] J. Conlisk. Monotone Mobility Matrices. Journal of Mathematical Sociology 15 (1990), 173-191.

[6] V. Dardoni. Monotone Mobility Matrices and Income Distribution. Social Choice and Welfare 12 (1995), 181-192.

[7] S.N. Ethier, Th. G. Kurtz. Markov Processes - Characterization and Convergence. Wiley Series in Probability and Mathematical Statistics, New York Chicester: Wiley 1986.

[8] N. Jacob. Pseudo-differential Operators and Markov Processes. Vol. I,II,III. London: Imperial College Press, 2001, 2002, 2005.

[9] O. Kallenberg. Foundations of Modern Probability. Second ed., Springer 2002.

[10] V. N. Kolokoltsov. Measure-valued limits of interacting particle systems with $k$-nary interactions I. Probab. Theory Relat. Fields 126 (2003), 364-394.

[11] V.N. Kolokoltsov. On Markov processes with decomposable pseudo-differential generators. Stochastics and Stochastics Reports 76:1 (2004), 1-44.

[12] V. N. Kolokoltsov. Nonlinear Markov Semigroups and Interacting Lévy Type Processes. Journ. Stat. Physics 126:3 (2007), 585-642.

[13] V. N. Kolokoltsov. Nonlinear Markov Processes and Kinetic Equations. Monograph. To appear in CUP, 2010.

[14] V. N. Kolokoltsov. The Lévy-Khintchine type operators with variable Lipschitz continuous coefficients generate linear or nonlinear Markov processes and semigroups. arXiv:0911.5688v1 [Math.PR] (2009). To appear in PTRF.

[15] G.Q. Lang. Stochastic monotonicity and positive correlations of a type of particle systems on Polish spaces (Chinese). Acta Math. Sinica (Chin. Ser.) 52:2 (2009), 309-314.

[16] E. Maasoumi. On Mobility. In: A. Ullah, D.E.A. Giles (Eds.) Handbook of Applied Economic Statistics. Marcel Dekker, New York, 1998, p. 119-175.

[17] A. Mijatovic, M. Pistorius. Continuously monitored barrier options under Markov processes. arXiv:0908.4028v1

[18] G. Samorodnitski and M. Taqqu. Stochastic mootonicity and slepian-type inequalities for infinitely divisible and stable random vectors. Ann. Probab 21:1 (1993), 143-160.

[19] Jie-Ming Wang. Stochastic Comparison and Preservation of Positive Correlations for Lévy-type processes. Preprint 2007.

[20] Y.H. Zhang Y. H. Sufficient and necessary conditions for stochastic comparability of jump processes. Acta Mathematica Sinica, English Series, 2000, 16:1 (2000), 99-102. 